\title{
3D Bioprinted Spheroidal Droplets for Engineering the Heterocellular Coupling between Cardiomyocytes and Cardiac Fibroblasts
}

\author{
Raven El Khoury $\mathbb{D}^{1,2}$ Naveen Nagiah, ${ }^{1,2}$ Joel A. Mudloff $\mathbb{D}^{1,2}{ }^{1,2}$ Vikram Thakur, ${ }^{3}$ \\ Munmun Chattopadhyay $\left(\mathbb{0},{ }^{3}\right.$ and Binata Joddar $\left(\mathbb{C}^{1,2,4}\right.$ \\ ${ }^{1}$ Inspired Materials \& Stem-Cell Based Tissue Engineering Laboratory (IMSTEL), The University of Texas at El Paso, El Paso, \\ TX 79968, USA \\ ${ }^{2}$ Department of Metallurgical, Materials, and Biomedical Engineering, M201 Engineering, The University of Texas at El Paso, \\ 500 W. University Avenue, El Paso, TX 79968, USA \\ ${ }^{3}$ Department of Molecular and Translational Medicine, Center of Emphasis in Diabetes and Metabolism, Texas Tech University \\ Health Sciences Center, 5001 El Paso Drive, El Paso, TX 79905, USA \\ ${ }^{4}$ Border Biomedical Research Center, The University of Texas at El Paso, 500 W. University Avenue, El Paso, TX 79968, USA
}

Correspondence should be addressed to Binata Joddar; bjoddar@utep.edu

Received 6 May 2021; Accepted 25 November 2021; Published 28 December 2021

Copyright (c) 2021 Raven El Khoury et al. Exclusive Licensee Beijing Institute of Technology Press. Distributed under a Creative Commons Attribution License (CC BY 4.0).

\begin{abstract}
Since conventional human cardiac two-dimensional (2D) cell culture and multilayered three-dimensional (3D) models fail in recapitulating cellular complexity and possess inferior translational capacity, we designed and developed a high-throughput scalable 3D bioprinted cardiac spheroidal droplet-organoid model with cardiomyocytes and cardiac fibroblasts that can be used for drug screening or regenerative engineering applications. This study helped establish the parameters for bioprinting and cross-linking a gelatin-alginate-based bioink into 3D spheroidal droplets. A flattened disk-like structure developed in prior studies from our laboratory was used as a control. The microstructural and mechanical stability of the 3D spheroidal droplets was assessed and was found to be ideal for a cardiac scaffold. Adult human cardiac fibroblasts and AC16 cardiomyocytes were mixed in the bioink and bioprinted. Live-dead assay and flow cytometry analysis revealed robust biocompatibility of the 3D spheroidal droplets that supported the growth and proliferation of the cardiac cells in the long-term cultures. Moreover, the heterocellular gap junctional coupling between the cardiomyocytes and cardiac fibroblasts further validated the 3D cardiac spheroidal droplet model.
\end{abstract}

\section{Introduction}

Despite the ease of forming and maintaining 2D cultures, some key limitations like failure to mimic the in vivo microenvironment and the absence of important cell-cell and cell-matrix interactions, signal transduction, proliferation, and differentiation potential are still a concern [1]. While $2 \mathrm{D}$ cultures are most commonly used in in vitro research, they are inadequate in fully mimicking the native myocardium and its vascularization, preventing the identification of potential therapeutic targets for cardiovascular diseases [2]. Technical challenges in terms of controlled cell distribution and vascular innervation make in vivo trans- plantation of tissue engineered constructs a rate-limiting step [3]. Three-dimensional (3D) additive manufacturing (AM) technologies offer an exceptional control for design of constructs with complex architectural facets especially in the case of 3D biological tissues.

$3 \mathrm{D}$ bioprinting is the process of adopting a complex architectural design as found in $3 \mathrm{D}$ tissues and translating it into a construct using AM techniques that utilizes biomaterial scaffolds, cells, and growth factors to produce engineered constructs that can serve as physiological replicates of their in vivo counterparts [4]. Different combinations of $3 \mathrm{D}$ bioprinting techniques and biomaterial scaffolds have been used for tissue engineering overall [5]. 3D in vitro 
multicell systems can not only mimic and represent the in vivo environment $[5,6]$ but can also serve as a promising alternative to animal models to study cardiac developmental biology and recapitulate disease [7]. 3D spheroidal droplets are considered to be the most widely accepted models for $3 \mathrm{D}$ in vitro culture [8-10]. A scaffold with a spheroidal design is expected to provide an enhanced habitat for tissue formation as it enables sufficient distribution of oxygen, media, growth factors, nutrients, and ions into the scaffold for maintaining cell growth and proliferation [11].

In recent published work, our laboratory has robustly demonstrated the potential of naturally derived hydrogels including gelatin, alginate, and collagen as naturally derived biodegradable materials for bioprinting multilayered systems [12-18]. Sodium alginate, primarily derived from seaweed and exhibiting ionotropic cross-linking mechanism with calcium chloride, sustains bioprinted cell morphology and their functions $[11,19]$. The combination of sodium alginate with gelatin improves the ease of printing and its ability for cell anchorage due to the presence of cell adhesion moieties like arginylglycylaspartic acid- (RGD-) containing peptides [20,21]. Recent studies have also shown the supporting effects of these polymers on sustained release of extracellular matrix (ECM) proteins and growth factors secreted by the cells [22-24].

In this study, we implemented a unique droplet-based extrusion printing technique to 3D bioprint cardiac spheroidal droplets using a CELLINK bioprinter which was scaled up to a higher throughput 96-well array fabrication using the Advanced BioAssemblyBot (BAB), equipped with a sixaxis robotic arm. The printing parameters for developing these bioprinted cell 3D spheroid models were optimized, and the printed constructs were physically characterized for their mechanical integrity and structural stability through rheological and swelling studies. Cardiomyocytes and cardiac fibroblasts were premixed in the hydrogels and bioprinted to generate $3 \mathrm{D}$ cardiac spheroidal droplets. The resultant cell-based droplets were analyzed for cell viability, and the onset of heterocellular coupling was quantified for up to 21 days in vitro [16]. Since the full extent of cardiomyocyte-fibroblast electrical coupling in the heart, the mechanisms of its regulation, and its importance in health and disease have garnered recent substantial interest [25], we propose that these 3D bioprinted cardiac spheroid models can potentially be used in studying and improving the role of heterocellular gap junctional coupling in the transfer of cardiomyogenic signals from the cells within the spheroidal droplets for drug screening or regenerative engineering applications. We further expect the bioprinted 3D spheroidal droplets to be more economical and serve as an enhanced high-throughput platform for functional cardiac assays compared to the flat multilayered systems established earlier in our laboratory $[12,13,16-18,22]$.

\section{Results}

2.1. Design, Morphology, and Consistency of 3D Spheroidal Droplets. Figure 1(a) shows the 3D printer used for high- throughput printing of the spheroidal droplets (Figure 1(b)). Figure 1(c) depicts the structural design adopted for printing the scaffolds. Figure 1(d) represents the 3D printed spheroidal droplets obtained from a gelatin- and sodium alginate-based bioink. The optimized printing parameters for obtaining stable scaffolds using the CELLINK and the BAB platforms are summarized in Table 1. The objective of the parameter optimization was to reduce the shear stress acting on the bioink to obtain maximum geometric accuracy $[13,15]$. The various parameters for $3 \mathrm{D}$ printing were modulated to achieve geometric accuracy by maintaining the extrusion pressure constant due to its inverse effect on cell viability. This optimization process for printing of 3D spheroids resulted in four distinct generations (Gen I-III) prior to yielding the desired 3D cardiac spheroids (Gen IV). Corresponding results for each generation are in Supplementary Table S1.

The partial ionic cross-linking of the sodium alginate in the hydrogel mixture with different concentrations of calcium chloride solution was found to be beneficial in obtaining structurally stable 3D bioprinted hydrogels. Gelatin was not cross-linked before printing; however, it is expected that the bioink mixture containing gelatin and alginate would lead to physical entanglements between the two polymeric chains resulting in electrostatic interactions leading up to partial ionic cross-linking between these two subunits (Supplementary Table S1 and Figure S1). Suspension of the 3D bioprinted spheroidal droplets in castor oil confirmed its morphological and structural stability as depicted in Figure 1(e). The aspect ratio of the 3D bioprinted hydrogel spheroidal droplets was maintained between varying batches. Figure 1(f) represents the aspect ratio of the 3D bioprinted spheroidal droplets (Gen IV) over three batches from a total of 108 samples suggesting its consistency and reproducibility of the spheroidal prints using the in-house developed printing technique.

2.2. Microstructural Analysis. Figures 2(a)-2(d) represent the microstructural cross section of the $3 \mathrm{D}$ spheroidal droplets ( $a, b)$ and flattened disks ( $c, d)$ performed using scanning electron microscopy (SEM). Both spheroidal droplets and flat disks exhibited a porous and interconnected network allowing their use as 3D cell culture models [26]. However, a significant difference in average pore diameters of the 3D bioprinted spheroidal droplets and flattened disks (controls) $(p<0.001)$ was observed and depicted in Figure 2(e). Porosity in a hydrogel scaffold plays an important role in cell survival, proliferation, and migration while pore interconnectivity influences cell ingrowth, vascularization, and nutrient diffusion for cell survival [27]. Engineered tissue requires pore sizes greater than $200 \mu \mathrm{m}$ for rapid vascularization and survival [28]. A significantly higher average pore diameter was observed for $3 \mathrm{D}$ bioprinted hydrogel spheroidal droplets with respect to the control samples $(p<0.001)$. The average pore diameter of the 3D spheroidal droplet was $228.67 \pm 92.07 \mu \mathrm{m}$ while the controls exhibited an average pore diameter of $63.02 \pm 29.3 \mu \mathrm{m}(p<0.001)$. A higher pore diameter contributes to a significantly higher porosity thereby increasing the interconnectivity between 


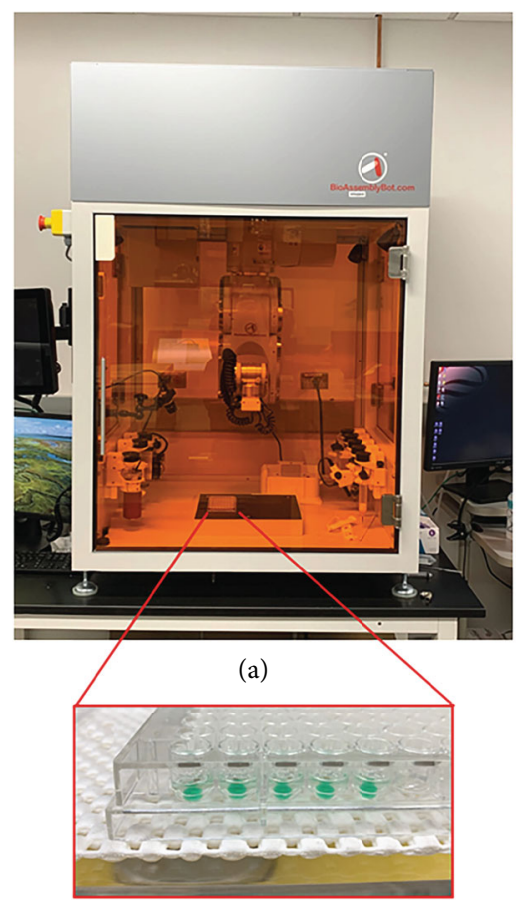

(b)

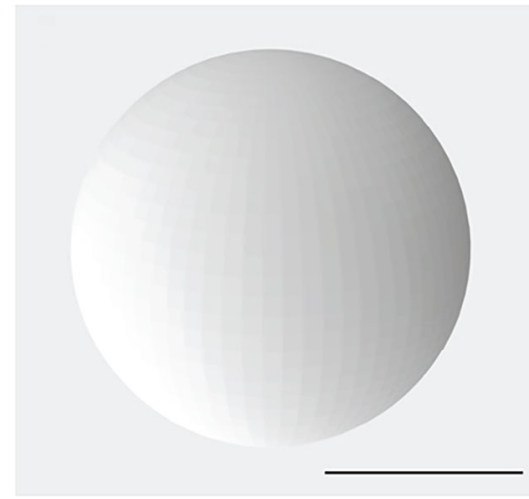

(c)

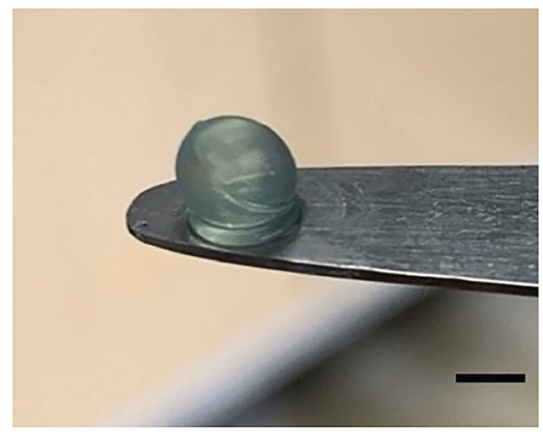

(d)

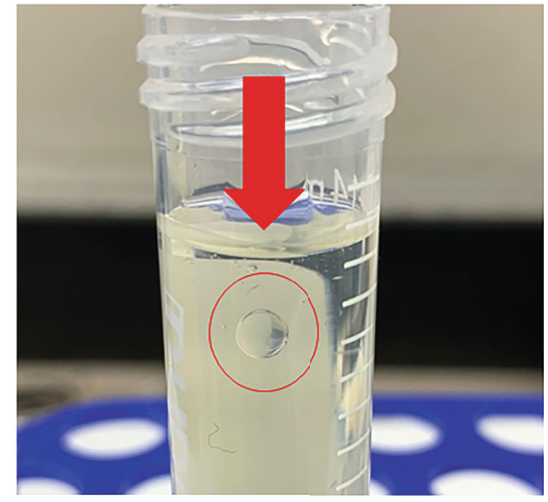

(e)

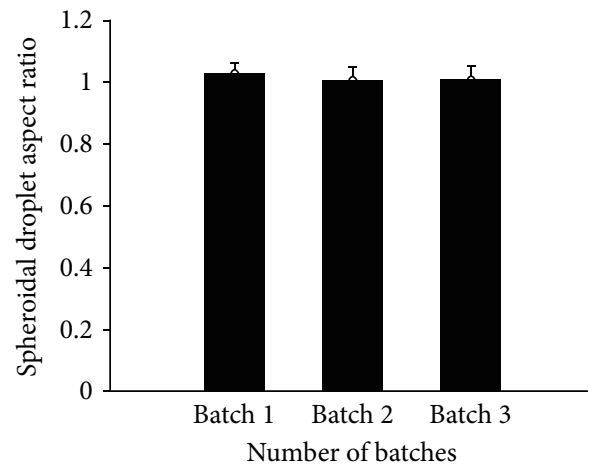

(f)

FIGURE 1: Design, morphology, and consistency of 3D spheroidal droplets. (a) The BioAssemblyBot (BAB) 3D printer used in bioprinting spheroidal hydrogels in a 96-well plate as shown in (b). (c) .stl file image of a spheroidal droplet. (d) Gross morphology of the 3D bioprinted spheroidal droplet. (e) Representative image showing a 3D bioprinted spheroidal droplet freely suspended in oil. (f) Aspect ratio is maintained for three consecutive 3D bioprinted batches. Scale bar was set at $1 \mathrm{~mm}$ for (c) and (d).

the pores which may enable and facilitate higher cell migration and diffusion of nutrients, supplements, and oxygen more efficiently in the 3D spheroidal droplets compared to the control structures $[12,29]$. Interestingly, this also sheds light on the possible mechanisms of the droplet printing in comparison with flattened disks. Since the 3D spheroidal droplets are extruded as a single droplet deposited within one well and cross-linked instantaneously, this leads to scaffolds with high porosity that are associated with higher mass transport of oxygen and nutrients, facilitating tissue regeneration [30]. On the other hand, the flattened disks have decreased average pore size and are associated with an increase in the surface area and suffer from decreased mass transport of oxygen and nutrients as well.

\subsection{Swelling and Mechanical and Structural Properties of} Bioprinted Constructs. Figure 3(a) shows the degree of swelling $\left(D_{s}\right)$ of the $3 \mathrm{D}$ spheroidal droplets in comparison to the flat disk control samples. 3D bioprinted hydrogel spheroidal droplets had a significantly higher degree of swelling that was nearly twice compared to controls $(p<0.01)$. For both samples, the maximum swelling ratio was observed after 24 hours from when samples remained stable throughout the duration of the experiment up to 28 days. The higher surface to volume ratio combined with the higher average pore diameter and open-closed pore ratio in 3D spheroidal droplets contributed to increased swelling compared to controls [31]. It is to be noted that the spheroidal droplets were stable till 28 days in 1x PBS and did not physically disintegrate while the control samples started physically disintegrating after 14 days. This confirms that the 3D spheroidal droplets can be utilized for long-term in vitro cell culture studies.

Figures 3(b)-3(d) represent the viscoelastic properties of the 3D spheroidal droplets and the control samples. While both sets of samples exhibited mainly elastic properties, the storage and loss modulus of the 3D spheroidal droplets and the control samples varied significantly as shown in Figure 3(b) $(p<0.001$ between the spheroidal droplets and controls). The average storage/loss modulus for the $3 \mathrm{D}$ spheroidal droplets was $3.84 \pm 0.38 / 0.597 \pm 0.054 \mathrm{kPa}$ while control structures exhibited $1.78 \pm 0.05 / 0.188 \pm 0.002 \mathrm{kPa}$ for storage and loss moduli, respectively. Similar to the storage and loss modulus, the elastic modulus of $3 \mathrm{D}$ bioprinted hydrogel spheroidal droplets was significantly higher than the control samples $(p<0.001)$. The storage modulus represents the energy stored in the elastic structure of the sample whereas the loss modulus represents the viscous part or the amount of energy dissipated in the sample $[32,33]$. If 


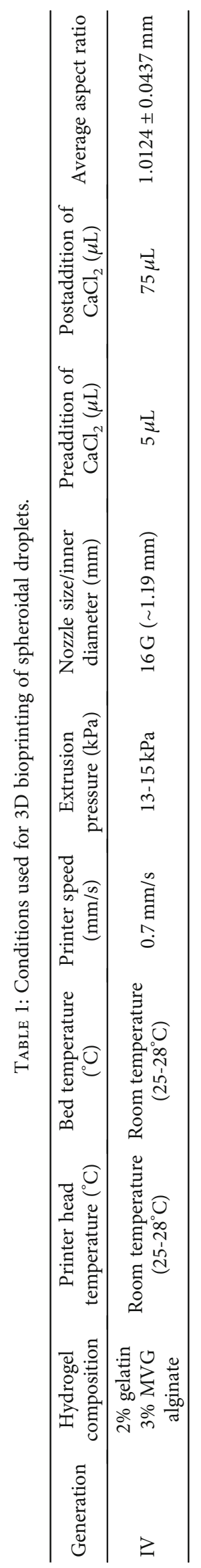



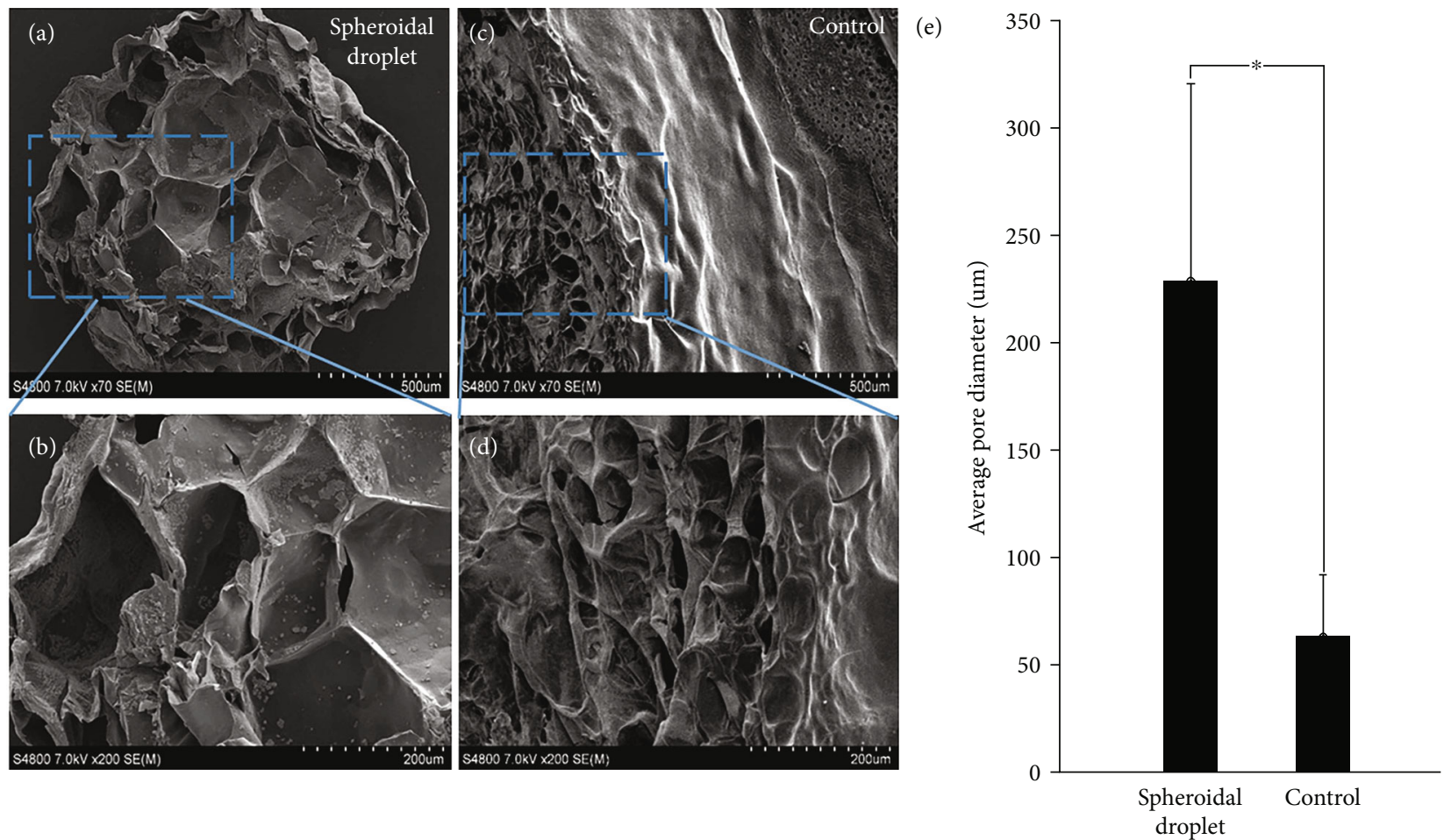

Figure 2: Microstructural analysis. (a, b) Representative SEM images for the cross section of the spheroidal droplets. (c, d) Representative SEM images for the cross-sectional structures of the flattened disk structure (control). (e) Graph depicting average pore diameter of the spheroidal droplet in comparison to the control structure. At least 5 representative images were acquired for analysis to generate the data in (e). Average pore diameter was found to be statistically different $\left({ }^{*} p<0.05\right)$ between the spheroidal droplet and control samples.

storage modulus is higher than the loss modulus, the material can be regarded as mainly elastic and vice versa. The elastic modulus, which is a measure of the scaffold's resistance to being deformed elastically, was also studied. The higher pore diameter and interconnectivity of the 3D spheroidal droplets in comparison with the controls may have manifested in higher sets of values for storage/loss and elastic moduli [25]. Thus, the degree of elasticity was greater in the $3 \mathrm{D}$ spheroidal droplets in comparison with the controls. The elastic modulus of the spheroidal scaffolds is within the range of the elastic modulus of native heart tissue $(>10-15 \mathrm{kPa})$ making it an attractive platform to be used as 3D cardiac organoids for in vitro studies. Although this data does not recapitulate values with cell content, it is estimated that inclusion of cells would yield higher values of elastic moduli which would pose as excellent choices for cardiac scaffolds used in tissue engineering.

Complex viscosity, which is a measure of the total resistance to flow as a function of angular frequency [34], was measured in both 3D spheroidal droplets and controls (Figure 3(c)). The 3D spheroidal droplets had a significantly higher complex viscosity (Figure 3(c)) $(309.78 \pm 30.86 \mathrm{~Pa} \cdot \mathrm{s})$ than controls $(143.11 \pm 8.58 \mathrm{~Pa} \cdot \mathrm{s})$.

2.4. Cell Viability within the Spheroidal Droplets. Figures 4(a) and 4(b) represent the live-dead confocal microscopy images of cells in the 3D spheroidal droplets after 7 and 14 days, respectively. More than $90 \%$ of cell viability was detected through the live-dead assay performed in the crosssectional core of the scaffold. Figure 4(c) shows no significant difference in the $\%$ of live cell viability between the two time points representing the steady turnover of the growing number of the cells in both scaffolds, spheroidal droplets and flat disks. Since no significant change in cell viability was noted at increasing time points, the mechanical stability of the scaffolds was robust enabling the viability of the cells. Live-dead images from other controls are depicted in Supplementary Figure 2.

In order to confirm if a necrotic core was formed in the center of the 3D spheroidal droplets containing cells, SEM and TUNEL assay was performed en face on the cross section of samples cut through the center at day 7 to corroborate the viability data obtained from imaging (as in Figure 4). Supplementary Figures $3 \mathrm{~A}$ and $3 \mathrm{~B}$ represent the SEM images of the 3D cell spheroidal droplets, and Supplementary Figure 3C represents the elemental analysis of the scaffolds with and without cells. The SEM images (S3A-B) revealed the encapsulation and distribution of cells within the spheroidal droplets. Moreover, we identified and located multiple possibly viable cells within the spheroidal structure, as seen from their adherence to the scaffold (yellow arrows in S3A). The elemental analysis obtained through energy-dispersive spectra (EDS) of the cell laden and acellular scaffolds shows a significant difference in $\mathrm{C}: \mathrm{N}: \mathrm{O}$ ratio between acellular and cell-laden 3D bioprinted hydrogel scaffolds signifying the presence of cells in the spheroidal droplets. 


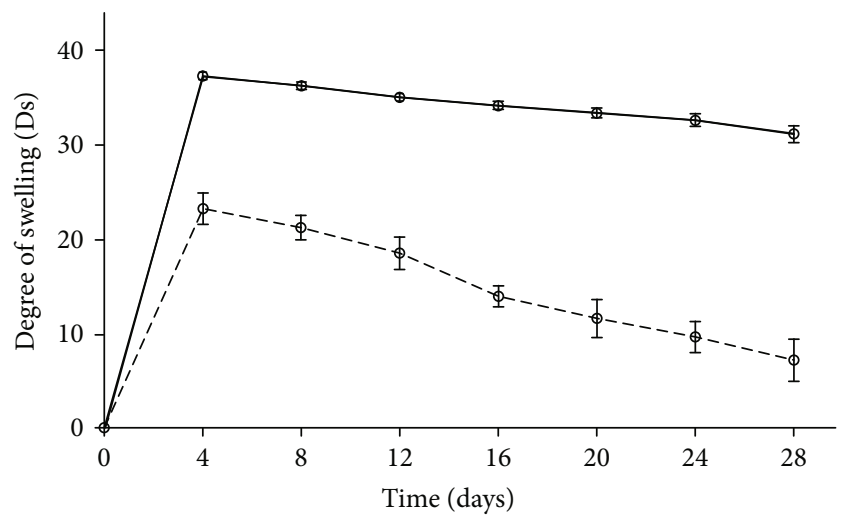

- Spheroidal droplet

-.-- Control

(a)

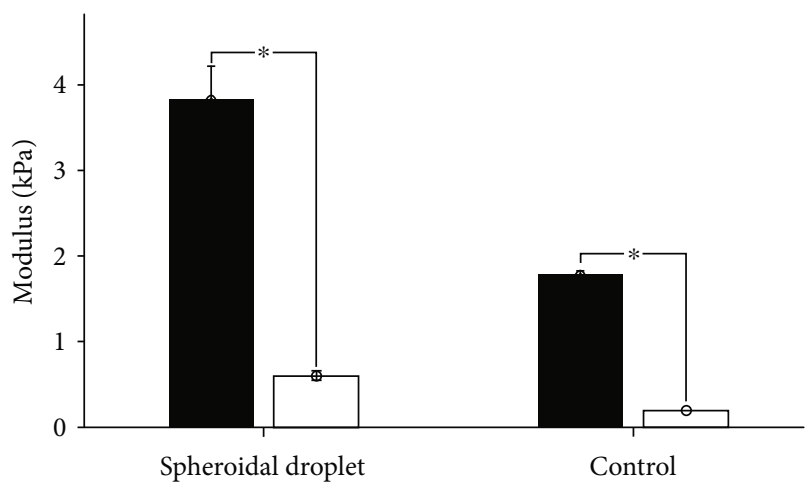

(b)

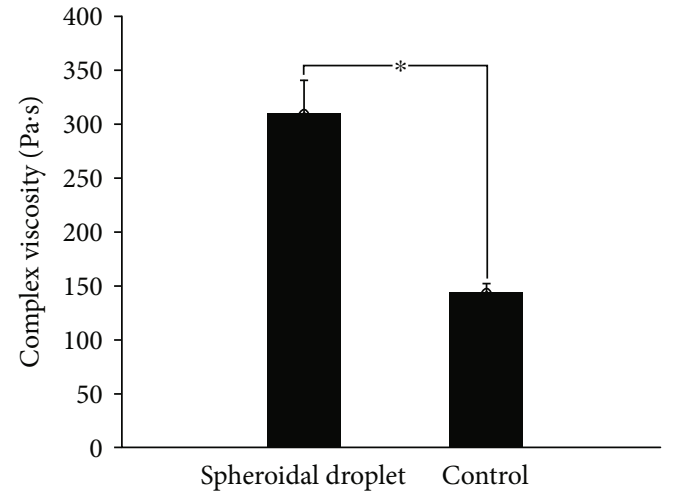

(c)

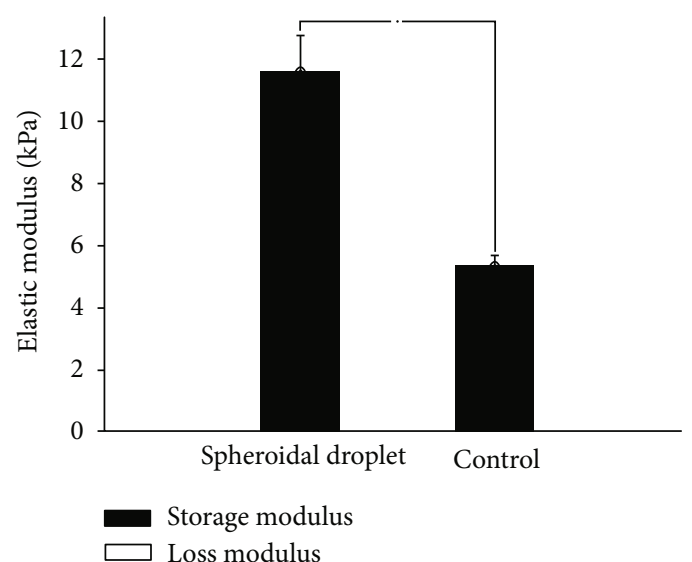

(d)

FIgURE 3: Mechanical characterization. (a) Graph depicting average degree of swelling (mean \pm SD) for spheroidal droplets (black) and controls (blue) for a period of 28 days. (b) Graph showing the storage modulus and loss modulus of the spheroidal droplet in comparison to control. (c, d) Graph showing the complex viscosity and the elastic modulus of the spheroidal droplet in comparison to control. At least three repeats $\left(n=3 ; 3 \times 24\right.$ spheroidal droplets and $3 \times 1$ for flattened disk) were used for calculation. ${ }^{*} p$ values were found to be all statistically different.

Furthermore, TUNEL assay results depicted in Figures 5(a)-5(c) confirm the absence of a necrotic core in the cardiac spheroidal droplets. When 3D cardiac spheroidal droplets cultured in vitro for 7 days were processed using the Click-iT $^{\mathrm{TM}}$ TUNEL Alexa Fluor ${ }^{\mathrm{TM}} 488$ tagged to the enzyme terminal deoxynucleotidyl transferase (TdT), no evidence of apoptotic DNA fragments could be detected (Figure 3(b)) when all cells were counterstained using Hoechst (Figure 3(a)). However, a parallel set of samples treated with ethanol for $5 \mathrm{~min}$ and then processed using the TUNEL assay revealed positive staining for the Alexa Fluor ${ }^{\mathrm{TM}} 488$ tagged to TdT (Figure 3(c)). This observation was corroborated also by the live/dead assay results in cells printed in a 3D spheroid stained in red by ethidium homodimer after intended apoptosis with ethanol treatment (Supplementary Figure S2C).
2.5. Heterocellular Coupling of CFs with CMs and Phenotypic Characterization. In an attempt to mimic in vivo cardiac tissue, the 3D spheroidal droplets must promote heterocellular coupling (HC) between cardiomyocytes and fibroblasts [22]. Representative high-magnification images of the $\mathrm{PKH}$ prestained CMs and CF in 3D spheroidal droplets after 14 days are depicted in Figures 6(a)-6(d). The cells confirmed their respective identities as $\mathrm{CM}$ and $\mathrm{CF}$ with heterocellular and cell junction coupling between the cells as shown in Figure 6(e) (top right corner). Troponin I (Trp-I: red) was used as a biomarker for staining the CM while fibroblastspecific protein I (FSP-I: green) was used as a biomarker for the CF. DAPI (blue) was used as an overall nuclear stain for all cells depicted in Figure 6. The enmeshing of both cell types, CM and CF (Figures 6(a)-6(d)), clearly confirmed their $\mathrm{HC}$ within the 3D spheroidal droplets at early and later 


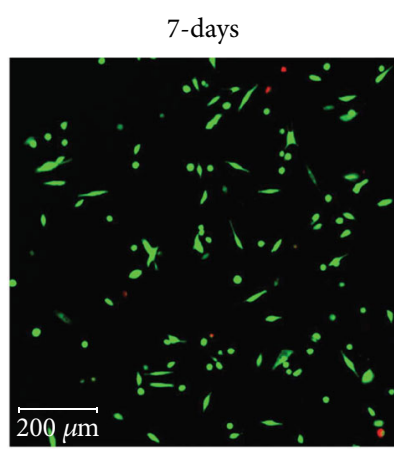

(a)

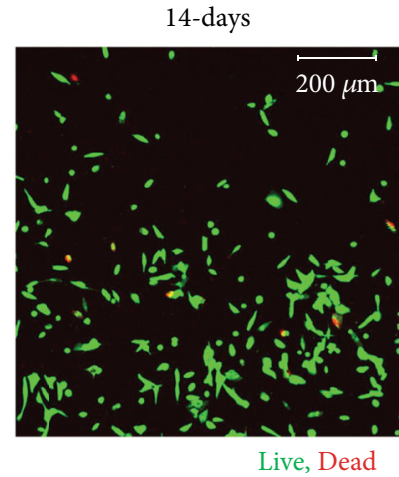

(b)

\% Live/ Dead cells

\begin{tabular}{|c|c|c|}
\hline & 7 -days & 14 -days \\
\hline Spheroidal Droplets & $98 \pm 2 / 5 \pm 2$ & $95 \pm 2 / 5 \pm 2$ \\
\hline Controls & $93 \pm 2 / 8 \pm 2$ & $96 \pm 2 / 10 \pm 4$ \\
\hline
\end{tabular}

(c)

Figure 4: Cell viability. (a, b) The live stained cells in green by calcein AM and dead cells stained in red by ethidium homodimer after 7 days and 14 days in culture (scale bar $200 \mu \mathrm{m}$ ). (c) \% live/dead cells in both 3D spheroidal droplets and controls at 7 and 14 days, respectively. $p$ values were found not to be statistically significant when data was compared between different time points.

time points in his study. The heterocellular coupling is consistent and maintained even after 21 days of culture as shown in Supplementary Figure S4. Figure 6(e) (bar graph) depicts the quantitative analysis of percentage $\mathrm{HC}$ between the 3D spheroidal droplets and controls (flattened disk images not included). The percentage of HC was $80.33 \pm 8.38 \%(p<0.05)$ for the $3 \mathrm{D}$ spheroidal droplets and $53.63 \pm 5.9 \%$ for controls further emphasizing the potential of using these $3 \mathrm{D}$ bioprinted spheroidal droplets as cardiac organoids. The extent of $\mathrm{HC}(\%)$ was confirmed to be within the range of studies published previously by our group [16].

Furthermore, Western blot analysis (as shown in Figure 6(e) bottom right corner) demonstrated a decrease in Trp-I $(24 \mathrm{kDa})$ expression in the controls in comparison to the spheroidal droplets $(p<0.05)$. This confirms the increased cell coupling and the presence of an increased density of cells in the spheroids which possibly leads to the enhanced expression of Trp-1 in these samples.

2.6. Cell Proliferation. We were specifically interested to study the proliferation trends for CM when they are coupled with the CF. Figures 7(a)-7(h) represent the flow cytometry analysis of cells encapsulated in the $3 \mathrm{D}$ spheroidal droplets. The CMs were stained using CellTrace ${ }^{\mathrm{TM}}$ Violet (CTV) dye, and the CFs were unstained. The CTV dye was used to track cell growth and proliferation using the concept of dye dilution and was used to investigate cell proliferation of CMs when coupled with $\mathrm{CF}$ in a 1:1 ratio in the $3 \mathrm{D}$ spheroidal droplets after 7 and 14 days of culture. This concept permits several generations of proliferating cells to be examined.
Thus, increasing cell proliferation causes a reduction in intensity of the dye over successive generations produced $[35,36]$. In this experiment, positive controls included CMs prestained with CTV and mixed with CF (1:1) for bioprinting within 3D spheroidal droplets and cultured for only 24 hours after which they were extracted. Negative controls included samples cultured using exact conditions as positive controls, without the addition of the CTV dye.

Figure 7 (a) represents characteristic peaks from positive controls wherein peaks for unstained CF are observed in the low-intensity region in the left $(<300)$ and peaks for stained $\mathrm{CM}$ with high intensity is observed in the right $(>300)$. The corresponding scatter plot for Figure $7(\mathrm{a})$ is depicted in Figure 7(b) where grey dots depict the CM and red dots depict the CF. Figure 7(c) represents characteristic peaks from negative controls where no cells were stained and shows peaks only in low-intensity regions in the left $(<300)$. The corresponding scatter plot for Figure $7(\mathrm{c})$ is depicted in Figure $7(\mathrm{~d})$. These characteristic peaks displayed by both sets of controls can be further explained by the higher intensity in the positive control $(74.6 \%)$ when compared to negative control $(12.43 \%)$ at day 1 . Figures $7(\mathrm{e})-$ 7 (h) represent the percentage (\%) of the dye intensity collected from samples extracted after 7 and 14 days of culture, respectively. The \% intensity of the CTV dye decreased from $67.17 \%$ at day 7 to $43.7 \%$ at day 14 . This reduction in intensity of the dye from the range $>300$ clearly signified the dilution of dye with time indicating proliferation of $\mathrm{CM}$ in the $3 \mathrm{D}$ spheroidal droplets. In addition, it is noteworthy to mention that the reason that the CM peaks were seen to be flattening out after 14 days of culture is because they 


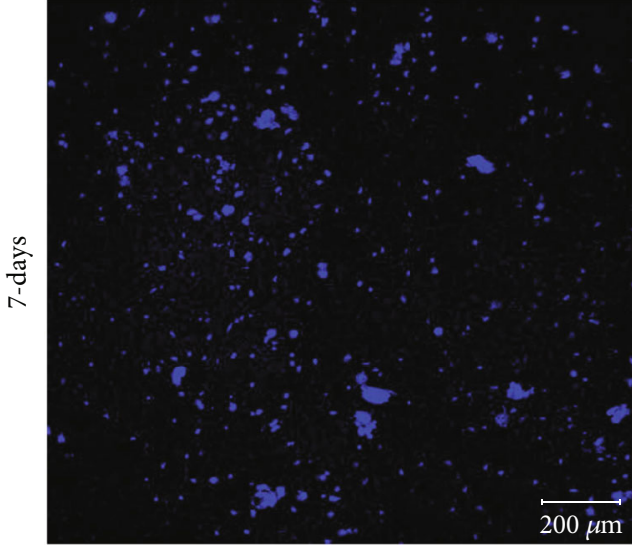

(a)

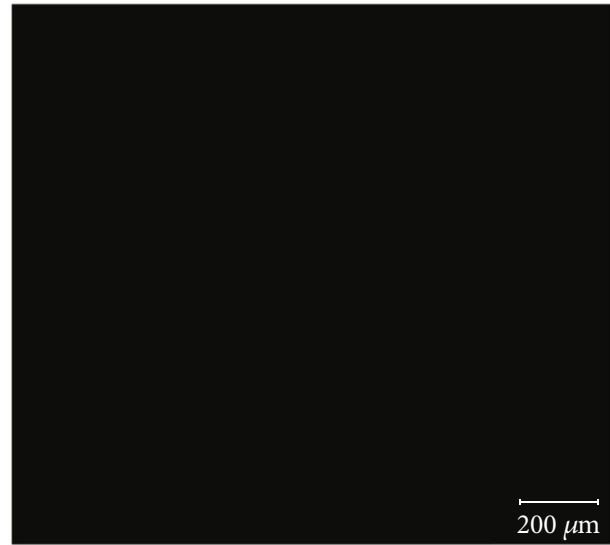

(b)

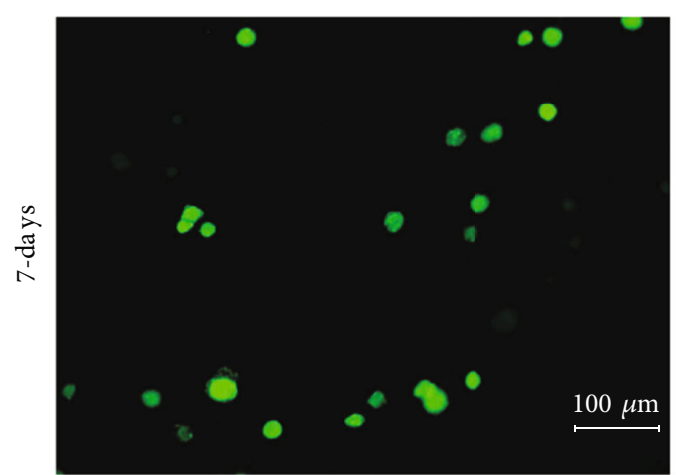

Hoechst, Click-iT ${ }^{\mathrm{TM}}$ TUNEL Alexa Flour ${ }^{\mathrm{TM}} 488$ tagged to TdT

(c)

FIGURE 5: TUNEL staining of 3D spheroidal droplets. (a) All the cells counterstained using Hoechst and (b) the absence of dead cells after 7 days in culture (scale bar $200 \mu \mathrm{m}$ ) imaged from the same sample section. (c) The presence of dead cells in the 3D spheroidal droplets.

were being outnumbered by CFs which grow more robustly and at a faster rate [37].

Moreover, the presence of multiple peaks (Figures $7(\mathrm{e})$ and $7(\mathrm{~g}))$ indicates the creation of new generations of cells during their incubation period. The corresponding scatter plots for Figures 7(e) and 7(g) are depicted in Figures 7(f) and $7(\mathrm{~h})$. All trends were statistically significant $(p<0.001)$.

Confocal microscopy images for CTV dye-stained cell samples are presented in the supplementary section (Figure S5).

\section{Discussion}

In recent years, 3D structures composed of multiple cells such as spheroidal droplets and organoids have been utilized extensively in in vitro research. Spheroidal droplets are 3D simple cell aggregates of broad-ranging cells, such as from tumor tissue [38], embryoid bodies [39], hepatocytes [40], nervous tissue [41], or mammary glands [42]. Cell-based clusters or cell spheroidal droplets are coaxed to form $3 \mathrm{D}$ cultures without a scaffold [38-42]. Thus, they cannot selfassemble or regenerate and thus are not as advanced as organoids. On the contrary, organoids are complex clusters of organ-specific cells and self-assemble when given a specific scaffolding extracellular environment, such as Matrigel [43] or collagen [44]. This leads to the development of microscopic versions of parent organs feasible for use as 3D study tissue models.

In this study, we developed a cardiac organoid model using spheroidal droplets for facilitating the heterocellular coupling between cardiac myocytes and cardiac fibroblasts. In the cardiac wall in vivo, cardiac myocytes and fibroblasts form extensive networks in the heart, with numerous structural contacts between these two cell types and other supporting cells. The cardiac fibroblasts secrete the majority of the extracellular cardiac tissue matrix, and their number increases with aging and during disease [45]. The cardiac myocytes which are coupled by connexin-43 (Cx43) gap junctions are known to be distinctly separated from fibroblasts in vivo. However, in vitro, these two heterogeneous cell types form functional gap junctions, which serve as a substrate for electrical coupling of cardiac myocytes [46]. Whether similar behavior occurs in vivo has been the subject of considerable discussion. Prior published studies from others including electrophysiological, immunohistochemical, and dye-coupling data confirmed the presence of direct electrical coupling between the two cell types in normal cardiac tissue in the sinoatrial node in vivo [47]. In our prior published studies, we have demonstrated proof of in vitro heterocellular coupling between cardiac myocytes 


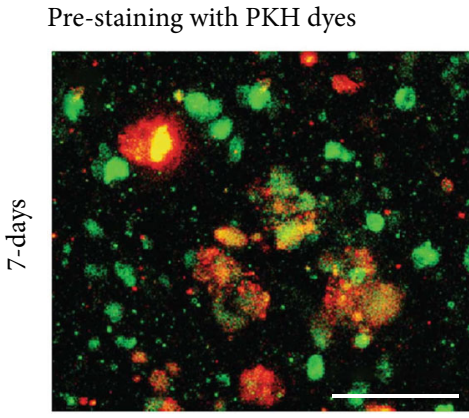

(a)
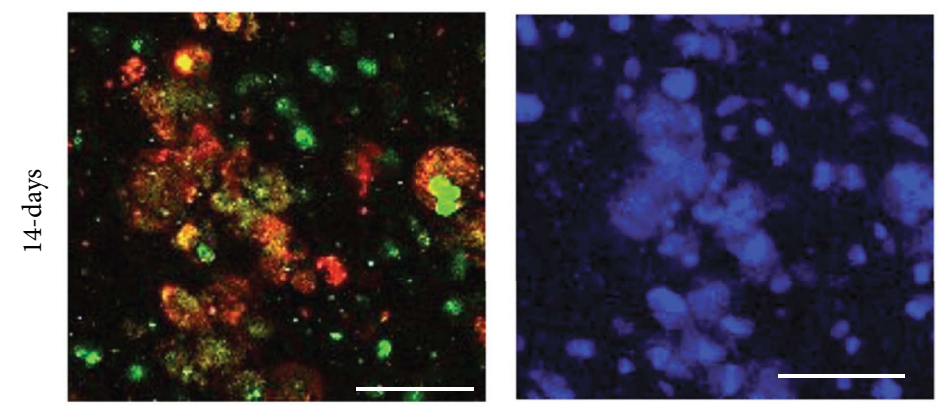

CM (PKH-67), CF (PKH26), DAPI

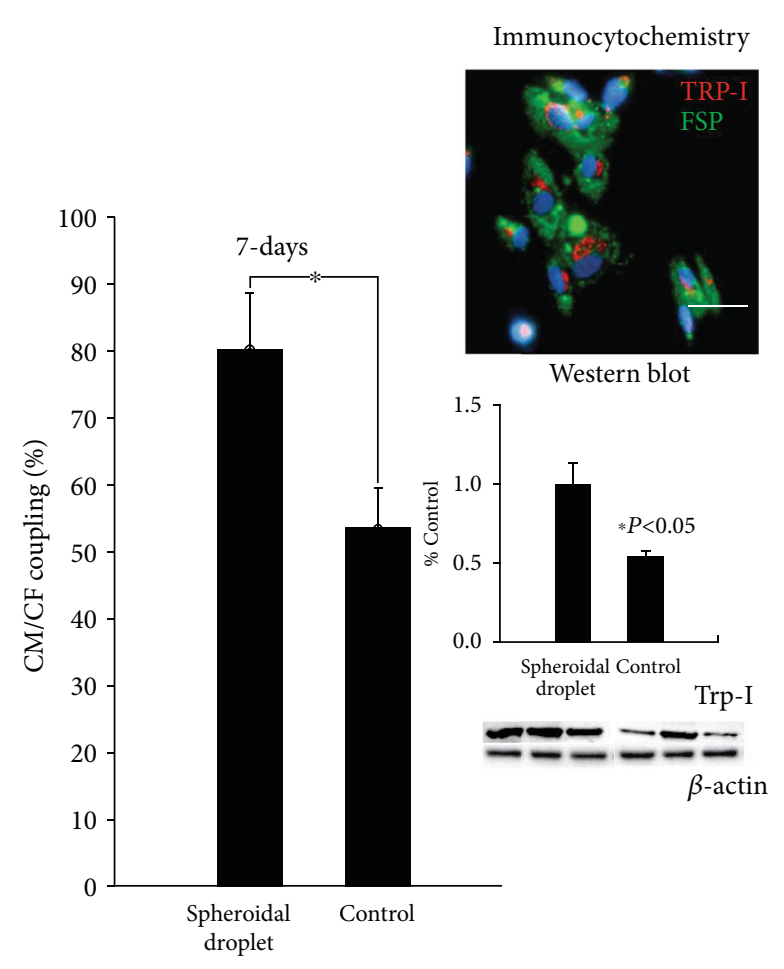

(e)

(c)

(d)

FIGURE 6: Confirmation of heterocellular coupling for cardiomyocyte (CM) and cardiac fibroblasts (CF) in the spheroidal droplets. Heterocellular coupling between CM (green) and CF (red) is shown after 7 and 14 days of culture (a-d). \% of heterocellular coupling is depicted using a bar graph in (e). The extent of coupling was found to be significantly greater in the $3 \mathrm{D}$ spheroidal droplets $(p<0.03)$ compared to the controls after 7 days of culture. Immunocytochemical data for CM-CF coculture at 7 days demonstrated the presence of $\mathrm{CM}$ and CF as analyzed by cell marker troponin I for CM and FSP for CF and imaged as shown in (e). Scale bar in all images is $100 \mu \mathrm{m}$. Furthermore, Western blot analysis demonstrated an increase in Trp-I expression in the spheroidal droplets compared to the controls, $p<0.05$. Trp-I bands for protein expression are depicted along with a row of $\beta$-actin that was used as a housekeeping control protein. Bands were obtained by running $25 \mu \mathrm{g}$ of protein, and membrane was developed using 1 min exposure.

and cardiac fibroblasts, facilitated by $3 \mathrm{D}$ bioprinting in vitro [16]. Thus, there is a strong correlation based on such heterogeneous cell coupling behavior, the cardiac electrical impulse conduction, and the transport of small molecules or ions in both the normal and pathological myocardium both in vitro and in vivo. Interestingly, cardiac fibroblasts have been shown to increase Cx43 expression in experimental models of myocardial infarction. Therefore, in the setting of cardiac disease, enhanced cardiac myocyte and fibroblast heterocellular coupling may influence the electrical activity of the myocytes and contribute to arrhythmias. This heterocellular coupling phenomenon is key to understanding the potential active contribution of nonmyocytes to cardiac electrophysiology and their relevance towards cardiac structure and function.

The key objective in this study was to create a $3 \mathrm{D}$ cardiac tissue model that may facilitate the study of biomarkers for targeting therapeutic strategies to treat cardiac disease and thereby allow for a better understanding of cardiac biology. We hypothesized that 3D cardiac cell spheroidal droplets will serve as a platform for enabling the heterocellular coupling between cardiac myocytes and fibroblasts for studying drug interactions in the future. After standardization of the
$3 \mathrm{D}$ bioprinting parameters, this study resulted in highthroughput production of $3 \mathrm{D}$ spheroidal droplets that exhibited interconnected porosity that promoted cell viability and function.

Each 3D spheroidal droplet has a volume of $4.18 \mathrm{~mm}^{3}$ and contains 20,000 \pm 1000 cells calculated from an initial cell density of 1 million cells per $\mathrm{mL}$ (corresponding to 4785 cells $/ \mathrm{mm}^{3}$ ). On the contrary, each control structure had a volume of $21.2 \mathrm{~mm}^{3}$ and contains $100,000 \pm 4000$ cells while maintaining cell density constant (corresponding to 4717 cells $/ \mathrm{mm}^{3}$ ). Furthermore, from $1 \mathrm{~mL}$ of bioink mixed with cells, approximately $45 \pm 3$ 3D spheroidal droplets versus $9 \pm 1$ flat structures can be printed. The possibility for further improvement of an already well-defined 3D biofabricated cardiac tissue system method was therefore established by showing that an advanced scaffold design may provide cellular diversity and culture efficiency [16]. Thus, the 3D cell spheroidal droplets represented a more efficient and economic model for studying cardiac cellular interactions [48-50]. This observation should remain consistent with any other 3D block structures if printed with cells for comparison with the spheroidal droplets. Furthermore, the spheroidal structures demonstrated a greater percentage of open 


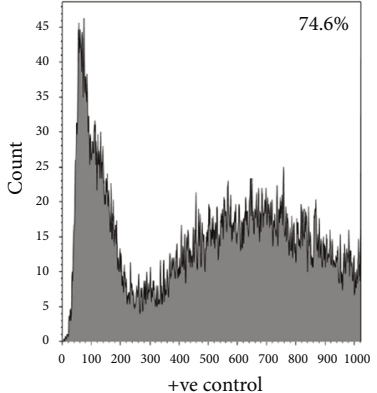

(a)

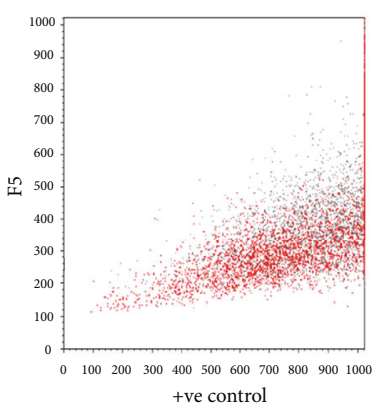

(b)

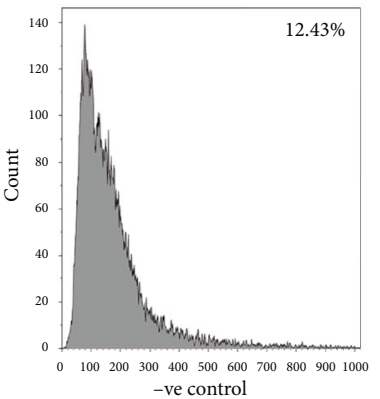

(c)

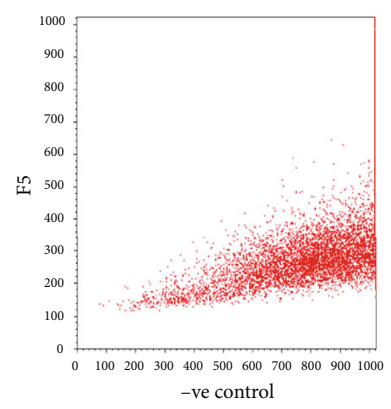

(d)

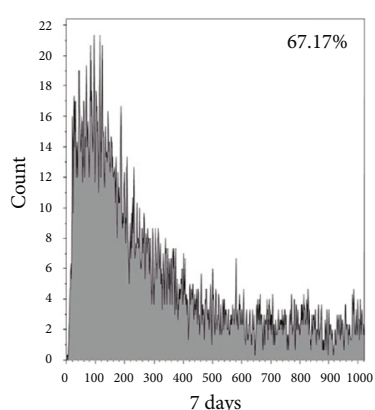

(e)

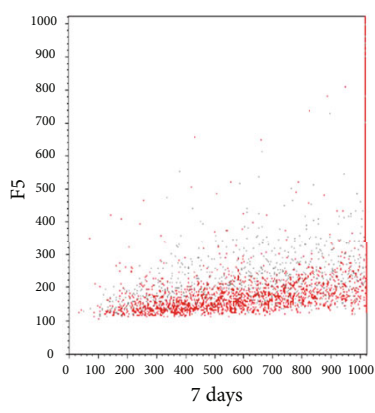

(f)

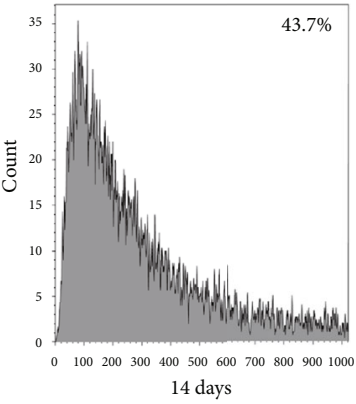

(g)

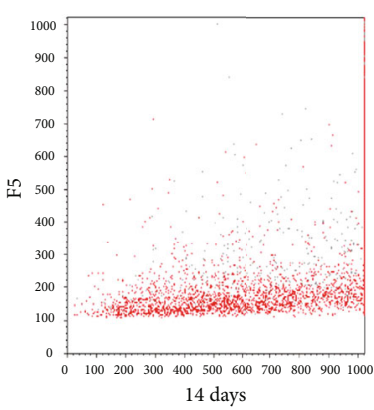

(h)

Figure 7: Cell proliferation analysis. CM cells were prestained with CellTrace Violet (CTV) and mixed with CF (1:1) for bioprinting within spheroidal droplets and cultured for long term in vitro. At 7 days and 14 days of culture, all cells were extracted from the spheroidal droplets and assessed using a FACS analyzer. Shown in $(\mathrm{a}, \mathrm{c}, \mathrm{e}, \mathrm{g})$ are representative graphs depicting $\%$ of CTV dye intensity (relates to the overall quantity of cells stained using CTV) for each time point and each condition. Positive control indicates CM cells prestained with CTV and mixed with CF $(1: 1)$ for bioprinting within spheroidal droplets and cultured for only 24 hours after which they were extracted. Negative control indicates the exact conditions for positive control without the addition of the CTV dye. Shown in (b, d, f, h) are the representative scatter plots showing the red dots (unstained CF) and grey dots (CTV-stained CM in all cases except -ve control) for each of the conditions depicted. In ( $\mathrm{a}, \mathrm{c}, \mathrm{e}, \mathrm{g})$, the $y$-axis depicts "counts," and in (b, d, f, h), the $y$-axis depicts "FS" or forward scatter plots.

versus closed pores in comparison to the flat disks. Interconnected networks of open pores are essential for cell nutrition, proliferation, and migration for new tissue formation [27]. Scaffolds with high resultant porosity (e.g., spheroids) enable effective transport of substrates for nutrient exchange. However, the mechanical fidelity of the spheroidal scaffolds was also maintained by a moderate presence of closed pores which lead to balance between the mechanical and mass transport function for an optimal scaffold system.

Like fetal cardiomyocytes, tissue engineered cardiac models also should express an upregulation and expression of cardiac troponin I (Trp-I) and tropomyosin as they transform into in mature cardiomyocytes [51]. Based on this hypothesis, we studied the expression levels of Trp-I in both 3D cell spheroidal droplets and 2D flattened disks. Our hypothesis was confirmed by the upregulation of Trp-I in the $3 \mathrm{D}$ cell spheroidal droplets in comparison to the $2 \mathrm{D}$ flattened disks.

The model geometry is significantly altered between the two structures, with the spheroidal droplets posing essentially as a 3D unit whereas the flattened disks posed as 2layered structures. Keeping gel volume constant for both structures, adoption of the two designs used in this study led to differences in degrees of curvature among both scaffolds. This led to alteration in cell behavior as well as also differences in mechanical behavior although both scaffolds were made using the same bioink [17].

Since homogenous delivery of oxygen to $3 \mathrm{D}$ static cell cultures remains an unsolved challenge, it can be expected that irregular oxygen supply may impede uniform cellular growth leading to decrease in overall cell viability within the spheroidal droplets over sustained periods, e.g., one month. In future studies, we will adopt dynamic culture conditions to sustain prolonged cell viability in long-term spheroidal cultures.

Our model can be used in high-throughput drug testing and screening processes allowing the automated testing of extensive and broad range of factors and biological compounds on a particular target or biomarker [52]. Nevertheless, the bioprinting protocol parameters and dimensions of the spheroid can be further adjusted to reflect a wider range of in vivo tissues and for usage in other applications such as tumor models $[53,54]$. On the other hand, drawbacks of such a model arise primarily from not resembling the striated structure of the native heart tissue [55]. Since the dimensions of the spheroid is such that it is made to fit inside a 96 well and since the maximum number of cells that it can hold is around $4 \times 10^{6} /$ sample at confluence, effective balancing of initial cell seeding density and scaffold volume must be taken into consideration prior to bioprinting. 
In the future, we will adopt this 3D spheroidal model for studying the contractile behavior of cell types such as HL-1 and even present a third cardiac cell type such as endothelial cells that will serve as a complete cardiac cell model applicable towards tissue engineering applications. The 3D spheroidal model is versatile and can be adopted for analysis of cell behavior and function to study the effect of various drugs that are known to impose cardiotoxicity $[56,57]$. This model is flexible enough to be adopted for other types of tissue engineering involving other cell types and can be used for microfluidic studies as well.

\section{Materials and Methods}

4.1. Chemicals and Cell Culture Reagents. Alginic acid (sodium salt; medium viscosity (MVG), MP Biomedicals, LLC, Illkirch, France) and gelatin type A (MP Biomedicals, LLC, OH, USA) were mixed together to form the hydrogel mixture. Sodium citrate and ethylenediaminetetraacetic acid (EDTA) were procured from Fisher Scientific (USA). Calcium chloride solution was created from calcium chloride dihydrate (Fisher Chemical, Germany) mixed with phosphate-buffered saline 10x solution (Fisher Bioreagents, USA) as an ionic cross-linker for the hydrogel scaffolds. Castor oil USP (Walgreen CO, Illinois, USA) was used to suspend the printed spheroidal droplets for structural analysis.

Cardiac fibroblast complete growth medium (Cell Applications, CA, USA) was used as such to culture CFs, and the CMs were cultured and expanded in Dulbecco's modified Eagle medium (DMEM/F12; Sigma Cat. No. D6434) containing $2 \mathrm{mM}$ L-glutamine (EMD Millipore Cat. No. TMS002-C), 12.5\% FBS (EMD Millipore Cat. No. ES-009-B), and $1 \mathrm{x}$ penicillin-streptomycin solution (EMD Millipore Cat. No. TMS-AB2-C). CellTrace Violet proliferation kit (Invitrogen, Carlsbad, CA) and DAPI (Thermo Fisher Scientific, USA) were used for cell labeling. In addition, 96 round-bottom well plates and 24 and 6 flat-bottom well plates (Thermo Fisher Scientific, USA) were used for bioprinting and cell culture, respectively, and Trypsin-EDTA (0.25\%, phenol red, Thermo Fisher) for cell detachment; 3-(4,5-dimethylthiazol-2-yl)-5-(3-carboxymethoxyphenyl)-2(4-sulfophenyl)-2H-tetrazolium (MTS; G3580, Cell Titer 96 Aqueous One Solution Cell Proliferation Assay, Promega, USA) was used for cell viability assays. The LIVE/DEAD ${ }^{\circledR}$ Viability/Cytotoxicity Kit was acquired from Thermo Fisher to image the extent of viability of cells. Multitissue dissociation kit 1 (Miltenyi Biotec GmbH, Bergisch Gladbach, Germany) was used to enzymatically digest the hydrogels for cell extraction and PFA (paraformaldehyde solution, $4 \%$ in PBS, Janssen Pharmaceuticals, Belgium) applied for cell fixation. Click-iT ${ }^{\text {TM }}$ Plus TUNEL Assay for In Situ Apoptosis Detection was acquired from Invitrogen ${ }^{\mathrm{TM}}$, Thermo Fisher Scientific, USA. Hoechst 33342 was used for counterstaining of cell nuclei (Sigma-Aldrich, St. Louis, MO, USA). Reagents for Western blotting are included in Section 4.10.

PKH26 Red and PKH67 Green, Fluorescent Cell Linker Kit for General Cell Membrane Labeling were acquired from Sigma-Aldrich.
4.2. Preparation of Alg/Gel Hydrogels. The bioink solution of $2 \% w / v$ gelatin was dissolved in Milli-Q water $\left(37^{\circ} \mathrm{C}\right)$ under constant stirring to which $3 \% w / v$ alginate was added under aseptic conditions [58]. The ink solution was next vortexed for $2 \mathrm{~min}$ to dissolve the alginate-gelatin mixture and centrifuged at $1200 \mathrm{rpm}$ for $3 \mathrm{~min}$ to remove the remaining air bubbles. Before cell printing, gels were UV sterilized for $15 \mathrm{~min}$ after which they were loaded into a $3 \mathrm{~mL}$ syringe (CELLINK, Blacksburg, VA, USA).

4.3. Fabrication of Bioprinted Constructs. A 3D spheroid of dimensions $2 \mathrm{~mm} \times 2 \mathrm{~mm}$ was designed using SolidWorks ${ }^{\circledR}$ software. The scaffold design was then converted to a binary .stl file using Meshmixer ${ }^{\circledR}$, a prototype designer tool. Using CELLINK BIO X (Blacksburg, VA, USA), the temperaturecontrolled printer head was used to deposit droplets inside a 96 round-bottom well plate. $5 \mu \mathrm{L}$ of $80 \mathrm{mM} \mathrm{CaCl}_{2}$ sterile solution was preadded to the bottoms of each of the wells. As shown in Table 1, air pressure was set between 13 and $15 \mathrm{kPa}$, speed selected to be $0.7 \mathrm{mms}^{-1}$, and a nozzle size of $16 \mathrm{G}(\mathrm{ID}=1.2 \mathrm{~mm} / \mathrm{OD}=1.6)$. After printing, alginategelatin composite scaffolds were cross-linked with an additional $75 \mu \mathrm{L}$ of $80 \mathrm{mM} \mathrm{CaCl}_{2}$ solution for $45 \mathrm{~min}$ under dynamic conditions while being placed on a Belly Dancer Shaker (IBI SCIENTIFIC, Iowa, USA). For highthroughput droplet printing of the hydrogel, BioAssemblyBot was used. An array of spheroidal droplets with identical dimensions was designed using TSIM software, and design file was transferred into the HMI software (Human Machine Interface) connected to the $\mathrm{BAB}$. High-throughput 3D bioprinting was achieved using parameters as mentioned before. A flattened disk was 3D printed using the same parameters [12], served as a control structure in this study. Scaffolds were cross-linked and washed under dynamic conditions with $1 \mathrm{x}$ PBS ( $\mathrm{pH}$ 7.4) for 20 minutes to remove excess calcium chloride. To test the uniformity and the conservation of the overall structure of the 3D spheroidal droplets, three distinct batches ( $n=36 /$ batch) were printed. Random samples picked from each of these three batches were then submerged in castor oil, and the aspect ratio of each spheroid sample was calculated using ImageJ by finding the relationship between the height and width of each corresponding spheroid generation [59] and depicted in

$$
\text { Aspect ratio }=\frac{\text { Height }}{\text { Width }} .
$$

4.4. Scanning Electron Microscopy. En face and crosssectional images of the previously lyophilized hydrogels were analyzed using a SEM (Hitachi, S-4800, Japan). Samples for imaging were sputter coated with gold for 45 seconds using Gatan Model 682 precision etching coating system, Pleasanton, CA, and imaged between 5 and $10 \mathrm{kV}[12,14]$. The elemental analysis of the cross section of 3D bioprinted hydrogel spheroidal droplets with/without cardiac cells was carried out in situ using the energy-dispersive X-ray spectroscopy (EDS) instrument in conjunction with the SEM. The characterization of elemental composition revealed the presence of $\mathrm{C}, \mathrm{O}, \mathrm{N}$, and $\mathrm{Na}$. The electron beam was focused 
on cells adhered to scaffold in the core and on the scaffolds alone in case of acellular scaffolds to show the difference in elemental composition as described by other published works [60]. An in-depth microstructural analysis was performed using ImageJ, and average (AVG) pore diameter was calculated utilizing

$$
\text { AVG Pore Diameter }=\frac{\sum \text { Pore Diameters }}{\text { Total No.of Pores }}
$$

Porous scaffolds have a mesh-like structure with open surface pores which are often interconnected in the bulk [61]. On the other hand, closed pores appear as cavities in the scaffolds but are not interconnected with the rest of the scaffold structure [62]. Scaffold porosity is determined by accounting for closed and open pores of varying size, shape, spatial distribution, and mutual interconnection. The greater extent of open porosity has a considerable influence on the physiochemical properties of the scaffold as well as biocompatibility and tissue regeneration.

4.5. Swelling and Degradation. The 3D spheroidal droplets and controls were maintained at $-80^{\circ} \mathrm{C}$ for 24 hours and lyophilized using BenchTop Pro with Omnitronics, SP Scientific, PA, USA $\left(-86^{\circ} \mathrm{C}, 205 \mathrm{mT}\right)$ for another 24 hours [12]. After freeze-drying, samples were weighed $\left(W_{0}\right)$ and immersed in $1 \mathrm{x}$ PBS. At various time points, the samples were weighed at room temperature $[12,15]$. Swelling ratio was calculated using the formula below for samples equilibrated in PBS for up to at least 28 days.

$$
D_{s}=\frac{W_{i}-W_{0}}{W_{0}} .
$$

4.6. Rheological Analysis. To examine the rheological properties of the experimental and control constructs, hydrogels were preswollen in $1 \mathrm{x}$ PBS for $24 \mathrm{~h}$ before testing. Oscillatory shear stress rheometric study was performed using an Anton-Paar MCR 92 rheometer (Anton-Paar, Austria) with a PP25/S measuring system at $1 \%$ strain with a frequency range between 0.5 and $50 \mathrm{~Hz}$ [63]. Analysis for frequency and strain was conducted within the viscoelastic range of the gels. Storage/loss moduli, complex viscosity, and elastic modulus were measured at $1.99 \mathrm{~Hz}$ as previously done and reported [12-14, 63].

4.7. Biocompatibility. Human cardiac fibroblasts (CF, adult; Cell Applications, San Diego, CA) and AC16 human cardiomyocyte or cardiac myocyte cell line (CM, ATCC, Manassas, VA) were mixed in a ratio of $1: 1$ to constitute a final cell seeding density of $1 \times 10^{6}$ cells added to $1 \mathrm{~mL}$ of the alginate-gelatin bioink solution and $3 \mathrm{D}$ bioprinted to obtain cell laden 3D bioprinted spheroidal droplets and flattened disks as controls. The ratio of $1: 1$ for mixing the cells was adopted from prior published studies wherein the ratio of these cells in the native cardiac tissue was revisited [64]. Cultures were incubated with a complete growth medium made from $\mathrm{CF}$ and $\mathrm{CM}$ growth media combined together in a ratio of $1: 1$ and maintained at $37^{\circ} \mathrm{C}$ and at $5 \% \mathrm{CO}_{2}$. To assess the biocompatibility of the 3D spheroidal droplets, Live/Dead Assay Kit (Thermo Fisher Scientific, USA) was used according to the protocol provided by the manufacturer. Calcein AM (green) represented live cells while ethidium homodimer (red) represented dead cells; viability of cells was quantified using

$$
\text { No.of live/dead cells }(\%)=\frac{\# \text { of green or red cells }}{\begin{array}{l}
\text { Total no.of green and red cells } \\
\times 100 .
\end{array}}
$$

To confirm their HC, biomarkers (see Section 4.9) were used in addition to prestaining the cells with $\mathrm{PKH}$ dyes, according to the protocol provided by the manufacturer. Briefly, the CMs were stained using PKH67 (green) and the CFs were stained using the PKH23 (red) as previously reported by our group [12-14].

The samples for HC analysis were fixed with PFA for $15 \mathrm{~min}$ at room temperature $\left(25-28^{\circ} \mathrm{C}\right)$ prior to imaging. The samples were then washed thrice with $1 \mathrm{x}$ PBS, mounted using Fluor mount-G with DAPI (Thermo Fisher Scientific). Using an LSM 700 confocal microscope system (Zeiss, Germany), Z-stack projection high-magnification images were acquired for each representative sample in each group and the experiment was repeated at least thrice. The average coupling percentage was obtained using

$$
\begin{aligned}
& \% \text { Coupling }=\frac{2 \times(\# \text { of coupled CMs }(\text { green }) \& C F s ~(\text { red })}{\text { Total no. of CFs }+ \text { CMs }} \\
& \times 100 .
\end{aligned}
$$

4.8. TUNEL Assay and Imaging. DNA fragmentation in the cells within the $3 \mathrm{D}$ bioprinted cardiac spheroidal droplets was determined using Click-iT ${ }^{\mathrm{TM}}$ Plus TUNEL Assay for In Situ Apoptosis Detection (Invitrogen ${ }^{\mathrm{TM}}$, Thermo Fisher Scientific, USA). After 7 days of culture, 3D spheroidal droplets were fixed in $4 \%$ paraformaldehyde for $15 \mathrm{~min}$ and then cut in half exposing the core of the scaffold followed by a staining procedure with picolyl azide Alexa Fluor ${ }^{\mathrm{TM}} 488$ visualizing the DNA degradation according to the manufacturer's protocol [65]. The nuclei were counterstained with Hoechst 33342 (Sigma-Aldrich, St. Louis, MO, USA). The stained sample sections were visualized using an LSM 700 confocal microscope system (Zeiss, Germany) using the appropriate filters. Z-stack projection high-magnification images were acquired for each representative sample in each group, and the experiment was done in triplicates.

4.9. Immunohistochemistry. The 3D bioprinted spheroid hydrogel constructs in media were harvested and fixed in $1: 1$ acetone/methanol fixative for 20 minutes at $-20^{\circ} \mathrm{C}$. This was followed by immunostaining using fibroblast surface protein (FSP-1 for CF, 1:400, Sigma), troponin I (for CM, $1: 400$, Thermo Fisher), and DAPI as mentioned previously to identify individual cell types [22, 49]. Goat anti-rabbit 
IgG secondary antibody-Alexa Fluor 594 or goat anti-mouse IgG secondary antibody-Alexa Fluor 488 conjugate applied at a dilution of $1: 1000$ was used as secondary antibodies. The samples were then washed thrice with $1 \mathrm{x}$ PBS, mounted using Fluor mount-G with DAPI (Thermo Fisher Scientific). Using a Nikon Eclipse NiE microscope system (Nikon Instruments Inc., Melville, NY), single-plane high-magnification images were acquired for each representative sample in each group and the experiment was repeated at least thrice [16]. For the \% coupling analysis, Z-stack images (3-5 images/sample) were used for the data calculation.

4.10. Western Blot. Samples isolated from culture were washed thrice with sterile 1x PBS until they were clear from culture media. Next, the samples were suspended in a solution of sodium citrate and EDTA $(1: 1)$ at final concentration of $0.05 \mathrm{M}$ each for $15 \mathrm{~min}$ at RT. After this step, the gels were cut apart using a cell scraper into smaller fragments and the suspension was homogenized with pipetting. Once the suspension was completely homogenized, it was centrifuged at $400 \times g$ for 10 min to collect the cells in a pellet form [66].

To the isolated cell pellet, $2 \mathrm{x}$ Laemmli buffer (Bio-Rad Laboratories, USA) containing protease inhibitor cocktail (5x) and dithiothreitol (DTT:100 mM) was added to resuspend the pellet and vortex it; following which the samples were heated at $100^{\circ} \mathrm{C}$ for $5 \mathrm{~min}$ to extract the cellular proteins. Next, the extracted proteins were estimated using a NanoDrop A280 (Thermo Fisher Scientific) that measures absorbance of mainly tryptophan and phenylalanine and provided an overall estimation of the amounts of amino acids in the protein mixture present in our extracts. A total of $25 \mu \mathrm{g}$ of protein was run in gels (Bolt 4-12\% BT Plus, Life Technologies) at $100 \mathrm{~V}$ for $2 \mathrm{~h}$. For the transfer, PVDF membranes of $40 \mu \mathrm{m}$ size were used to capture the proteins in a Mini Blot Module, Life Technologies) at $20 \mathrm{~V}$ for $60 \mathrm{~min}$.

After successful transfer of proteins, the membrane was blocked with $5 \%$ nonfat milk in $1 \mathrm{x}$ TBS-T, rinsed thrice with 1x TBS-T for 5 min each followed by antibody treatment. A primary antibody treatment using Trp-I (Life Technologies, 16A11) was done in a $1: 500$ dilution in $1 \mathrm{x}$ TBS-T for $1 \mathrm{~h}$ at RT, followed by three washes with TBS-T for $5 \mathrm{~min}$ each. Next, the membrane was probed with a secondary antibody solution of goat anti-mouse $\operatorname{IgG}(\mathrm{H}+\mathrm{L})$ Superclonal $^{\mathrm{TM}}$, HRP conjugate based on suppliers' protocols (Life Technologies, A28177) diluted $1: 4000$ in $1 \mathrm{x}$ TBS-T by $1 \mathrm{~h}$ at RT, washed thrice with TBS-T for $5 \mathrm{~min}$ each, and immediately imaged with Pierce ${ }^{\mathrm{TM}}$ ECL Western Blotting Substrate (Thermo fisher, PI32209). $\beta$-Actin $(1: 2000$; MilliporeSigma, St. Louis, $\mathrm{MO}$, USA) was used as a loading control, and the data were normalized with the respective level of $\beta$-actin using an image analysis software (ChemiDoc XRS System, Bio-Rad Laboratories, Hercules, CA, USA) to determine the intensity of each band ( $n=3$ sample per group) after which the data were further analyzed to assess the percent of control.

4.11. Flow Cytometry Analysis (FACS). Cardiomyocytes were prestained using CellTrace ${ }^{\mathrm{TM}}$ Violet (CTV) proliferation kit
(Invitrogen, Carlsbad, CA) according to the manufacturer's protocol prior to $3 \mathrm{D}$ bioprinting with the cardiac fibroblasts in alginate-gelatin hydrogel. To further confirm the uptake and staining of the CTV dye in cells prior to FACS analysis, cells were imaged using an EVOS bright field microscope (Figure S4). The 3D spheroidal droplets with cells were cut using a blade, and cells were extracted using Miltenyi gentleMACS Dissociator (Miltenyi Biotec, Cambridge, MA) using a Multitissue Dissociation Kit-1 by running the Multi_B program according to the manufacturer's protocol. After 7 and 14 days, cells were fixed with 4\% PFA for $15 \mathrm{~min}$ at room temperature and added to their designated FACS analysis falcon tubes and analyzed using Beckman Coulter Gallios Flow Cytometer (Brea, CA, USA) using excitation and emission wavelengths of 405 and $450 \mathrm{~nm}$, respectively.

4.12. Statistical Analysis. All experiments were performed in triplicate with varying passage of cells for both cardiomyocytes and cardiac fibroblasts (passages 8-12) and scaffolds (from at least 3 different batches) for this study, and numerical data are reported as mean \pm standard deviation. Each experiment included at least 3 technical replicates and 3 biological replicates. All data were compared using Student's $t$-test with $p<0.05$ considered to be statistically significant.

\section{Conflicts of Interest}

The authors declare no conflicts of interest regarding the publication of this article.

\section{Authors' Contributions}

All authors planned the experiments and contributed to the data analysis. REK performed the experiments under the mentoring of $\mathrm{NN}$ and supervision of BJ. JAM performed process development and optimization for the highthroughput printing of the droplets on a $\mathrm{BAB}$ platform. VT and MC helped grow the cells used in this study and contributed to the IHC and HC studies. REK, NN, and BJ wrote the manuscript and edited it. All authors have approved the final version for submission.

\section{Acknowledgments}

The Joddar laboratory (IMSTEL) acknowledges NSF grant/s \# 1828268 and \# 1927628 and NIH SC1 grant \#1SC1HL154511-01. The Chattopadhyay lab acknowledges NSF grant \# 1949909. JAM acknowledges support from National Institute of General Medical Sciences of the National Institutes of Health under RL5GM118969 and TL4GM118971 and the NIH BUILD Pilot grant \# 8UL1GM118970-02. We acknowledge the technical assistance received from Monica Delgado for cell culture, Armando Varela for confocal microscopy, and Michael Lyubchenko for his help with the SEM and EDS. Carla Loyola performed and analyzed the Western blots. All data generated or analyzed during this study are included in this published article (and its supplementary information files). 
The raw datasets used to generate all the figures generated during and/or analyzed during the current study are available from the corresponding author on reasonable request.

\section{Supplementary Materials}

Table S1: the evolutionary process in engineering a 3D bioprinted spheroid. Figure S1: average aspect ratio (mean $\pm \mathrm{SD}$ ) calculations for the other corresponding generations as enlisted in Table S1. Figure S2: cell viability was assessed by performing the live/dead assay on $2 \mathrm{D}$ structures after 4 days of culture. Figure S3: scanning electron microscopy and EDS for cellular encapsulation. Figure S4: shown is an image depicting heterocellular coupling between $\mathrm{CM}$ (green) and CF (red) after 21 days of culture (left). All cells $(\mathrm{CM}+\mathrm{CF})$ in the presented image are depicted with DAPI (blue) staining (right). Figure S5: shown is a bright field image of CTV dye-stained CM cells. (Supplementary Materials)

\section{References}

[1] N. Chaicharoenaudomrung, P. Kunhorm, and P. Noisa, "Threedimensional cell culture systems as anin vitroplatform for cancer and stem cell modeling," World journal of stem cells, vol. 11, no. 12, pp. 1065-1083, 2019.

[2] M. Qasim, F. Haq, M. H. Kang, and J. H. Kim, “3D printing approaches for cardiac tissue engineering and role of immune modulation in tissue regeneration," International Journal of Nanomedicine, vol. 14, pp. 1311-1333, 2019.

[3] C. Mandrycky, Z. Wang, K. Kim, and D. H. Kim, "3D bioprinting for engineering complex tissues," Biotechnology Advances, vol. 34, no. 4, pp. 422-434, 2016.

[4] J. Kim, J. S. Kong, W. Han, B. S. Kim, and D. W. Cho, “3D cell printing of tissue/organ-mimicking constructs for therapeutic and drug testing applications," International Journal of Molecular Sciences, vol. 21, no. 20, article 7757, 2020.

[5] M. Askari, M. Afzali Naniz, M. Kouhi, A. Saberi, A. Zolfagharian, and M. Bodaghi, "Recent progress in extrusion 3D bioprinting of hydrogel biomaterials for tissue regeneration: a comprehensive review with focus on advanced fabrication techniques," Biomaterials Science, vol. 9, no. 3, pp. 535-573, 2021.

[6] P. R. Buenzli, M. Lanaro, C. S. Wong et al., "Cell proliferation and migration explain pore bridging dynamics in $3 \mathrm{D}$ printed scaffolds of different pore size," Acta Biomaterialia, vol. 114, pp. 285-295, 2020.

[7] P. Sharma and C. Gentile, "Cardiac Spheroids as in vitro Bioengineered Heart Tissues to Study Human Heart Pathophysiology," Journal of Visualized Experiments, vol. 167, 2021.

[8] C. Zuppinger, "3D cardiac cell culture: a critical review of current technologies and applications," Frontiers in cardiovascular medicine, vol. 6, pp. 87-87, 2019.

[9] B. Pinto, A. C. Henriques, P. M. A. Silva, and H. Bousbaa, "Three-dimensional spheroids as in vitro preclinical models for cancer research," Pharmaceutics, vol. 12, no. 12, p. 1186, 2020.

[10] G. Lazzari, P. Couvreur, and S. Mura, "Multicellular tumor spheroids: a relevant $3 \mathrm{D}$ model for the in vitro preclinical investigation of polymer nanomedicines," Polymer Chemistry, vol. 8, no. 34, pp. 4947-4969, 2017.
[11] T. Pan, W. Song, X. Cao, and Y. Wang, "3D bioplotting of gelatin/alginate scaffolds for tissue engineering: influence of crosslinking degree and pore architecture on physicochemical properties," Journal of Materials Science \& Technology, vol. 32, no. 9, pp. 889-900, 2016.

[12] S. AnilKumar, S. C. Allen, N. Tasnim et al., "The applicability of furfuryl-gelatin as a novel bioink for tissue engineering applications," Journal of Biomedical Materials Research Part B, Applied Biomaterials, vol. 107, no. 2, pp. 314-323, 2019.

[13] M. Alonzo, S. A. Kumar, S. Allen et al., "Hydrogel scaffolds with elasticity-mimicking embryonic substrates promote cardiac cellular network formation," Progress in Biomaterials, vol. 9, no. 3, pp. 125-137, 2020.

[14] C. M. Cleetus, F. Alvarez Primo, G. Fregoso et al., "Alginate hydrogels with embedded $\mathrm{ZnO}$ nanoparticles for wound healing therapy," International Journal of Nanomedicine, vol. 15, pp. 5097-5111, 2020.

[15] M. Alonzo, E. Dominguez, F. Alvarez-Primo et al., "A comparative study in the printability of a bioink and 3D models across two bioprinting platforms," Materials Letters, vol. 264, article 127382, 2020.

[16] S. Anil Kumar, M. Alonzo, S. C. Allen et al., "A visible lightcross-linkable, fibrin-gelatin-based bioprinted construct with human cardiomyocytes and fibroblasts," ACS Biomaterials Science \& Engineering, vol. 5, no. 9, pp. 4551-4563, 2019.

[17] S. Anil Kumar, N. Tasnim, E. Dominguez et al., "A comparative study of a $3 \mathrm{D}$ bioprinted gelatin-based lattice and rectangular-sheet structures," Gels, vol. 4, no. 3, p. 73, 2018.

[18] M. Alonzo, S. AnilKumar, B. Roman, N. Tasnim, and B. Joddar, "3D bioprinting of cardiac tissue and cardiac stem cell therapy," Translational Research, vol. 211, pp. 64-83, 2019.

[19] X. Zhang and Y. Zhang, "Tissue engineering applications of three-dimensional bioprinting," Cell Biochemistry and Biophysics, vol. 72, no. 3, pp. 777-782, 2015.

[20] M. Akhmanova, E. Osidak, S. Domogatsky, S. Rodin, and A. Domogatskaya, "Physical, spatial, and molecular aspects of extracellular matrix of in vivo niches and artificial scaffolds relevant to stem cells research," Stem Cells International, vol. 2015, Article ID 167025, 35 pages, 2015.

[21] J. Lewicki, J. Bergman, C. Kerins, and O. Hermanson, “Optimization of $3 \mathrm{D}$ bioprinting of human neuroblastoma cells using sodium alginate hydrogel," Bioprinting, vol. 16, article e00053, 2019.

[22] S. A. Kumar, M. Delgado, V. E. Mendez, and B. Joddar, "Applications of stem cells and bioprinting for potential treatment of diabetes," World Journal of Stem Cells, vol. 11, no. 1, pp. 13-32, 2019.

[23] A. Mondal, A. Gebeyehu, M. Miranda et al., "Characterization and printability of sodium alginate -gelatin hydrogel for bioprinting NSCLC co-culture," Scientific Reports, vol. 9, no. 1, article 19914, 2019.

[24] Z. Li, S. Huang, Y. Liu et al., "Tuning alginate-gelatin bioink properties by varying solvent and their impact on stem cell behavior," Scientific Reports, vol. 8, no. 1, article 8020, 2018.

[25] S. Bindgen, F. Bossler, J. Allard, and E. Koos, "Connecting particle clustering and rheology in attractive particle networks," Soft Matter, vol. 16, no. 36, pp. 8380-8393, 2020.

[26] J.-Y. Dewavrin, N. Hamzavi, V. P. W. Shim, and M. Raghunath, "Tuning the architecture of three-dimensional collagen hydrogels by physiological macromolecular crowding," Acta Biomaterialia, vol. 10, no. 10, pp. 4351-4359, 2014. 
[27] S. J. Hollister, "Porous scaffold design for tissue engineering," Nature Materials, vol. 4, no. 7, pp. 518-524, 2005.

[28] N. Annabi, J. W. Nichol, X. Zhong et al., "Controlling the porosity and microarchitecture of hydrogels for tissue engineering," Tissue Engineering. Part B, Reviews, vol. 16, no. 4, pp. 371-383, 2010.

[29] Q. L. Loh and C. Choong, "Three-dimensional scaffolds for tissue engineering applications: role of porosity and pore size," Tissue Engineering Part B, Reviews, vol. 19, no. 6, pp. 485502, 2013.

[30] C. M. Murphy and F. J. O’Brien, “Understanding the effect of mean pore size on cell activity in collagen-glycosaminoglycan scaffolds," Cell Adhesion \& Migration, vol. 4, no. 3, pp. 377$381,2010$.

[31] R. Blystone, "WWW.Cell biology educationCell Biology Education," vol. 3, no. 1, pp. 11-14, 2004.

[32] S. Patra, P. M. Ajayan, and T. N. Narayanan, "Dynamic mechanical analysis in materials science: the novice's tale," Oxford Open Materials Science, vol. 1, no. 1, 2020.

[33] M. Herrero-Herrero, J. A. Gómez-Tejedor, and A. VallésLluch, "Role of electrospinning parameters on poly(lacticco-glycolic acid) and poly(caprolactone-co-glycolic acid) membranes," Polymers, vol. 13, no. 5, p. 695, 2021.

[34] S. K. Giri, M. K. Tripathi, and N. Kotwaliwale, "Effect of composition and storage time on some physico-chemical and rheological properties of probiotic soy-cheese spread," Journal of Food Science and Technology, vol. 55, no. 5, pp. 1667-1674, 2018.

[35] J. Begum, W. Day, C. Henderson et al., "A method for evaluating the use of fluorescent dyes to track proliferation in cell lines by dye dilution," Cytometry Part A, vol. 83, no. 12, pp. 10851095, 2013.

[36] A. Filby, E. Perucha, H. Summers et al., "An imaging flow cytometric method for measuring cell division history and molecular symmetry during mitosis," Cytometry Part A, vol. 79, no. 7, pp. 496-506, 2011.

[37] J. Zhang, R. Tao, K. F. Campbell et al., "Functional cardiac fibroblasts derived from human pluripotent stem cells via second heart field progenitors," Nature Communications, vol. 10, no. 1, p. 2238, 2019.

[38] R. J. Porter, G. I. Murray, and M. H. McLean, "Current concepts in tumour-derived organoids," British Journal of Cancer, vol. 123, no. 8, pp. 1209-1218, 2020.

[39] M. Simunovic and A. H. Brivanlou, "Embryoids, organoids and gastruloids: new approaches to understanding embryogenesis," Development (Cambridge, England), vol. 144, no. 6, pp. 976-985, 2017.

[40] H. Hu, H. Gehart, B. Artegiani et al., "Long-term expansion of functional mouse and human hepatocytes as 3D organoids," Cell, vol. 175, no. 6, pp. 1591-1606.e19, 2018.

[41] R. Vieira de Sá, M. Cañizares Luna, and R. J. Pasterkamp, "Advances in Central Nervous System Organoids: A focus on organoid-based models for motor neuron disease," Tissue Engineering Part C: Methods, vol. 27, no. 3, pp. 213-224, 2021.

[42] P. R. Jamieson, J. F. Dekkers, A. C. Rios, N. Y. Fu, G. J. Lindeman, and J. E. Visvader, "Derivation of a robust mouse mammary organoid system for studying tissue dynamics," Development, vol. 144, no. 6, pp. 1065-1071, 2017.

[43] M. T. Pham, K. M. Pollock, M. D. Rose et al., "Generation of human vascularized brain organoids," Neuroreport, vol. 29, no. 7, pp. 588-593, 2018.
[44] J. H. Jee, D. H. Lee, J. Ko et al., "Development of collagenbased 3D matrix for gastrointestinal tract-derived organoid culture," Stem Cells International, vol. 2019, Article ID 8472712, 15 pages, 2019.

[45] C. Humeres and N. G. Frangogiannis, "Fibroblasts in the infarcted, remodeling, and failing heart," JACC Basic to translational science, vol. 4, no. 3, pp. 449-467, 2019.

[46] P. Kohl and R. G. Gourdie, "Fibroblast-myocyte electrotonic coupling: does it occur in native cardiac tissue?," Journal of Molecular and Cellular Cardiology, vol. 70, no. 100, pp. 3746, 2014.

[47] S. D. Unudurthi, R. M. Wolf, and T. J. Hund, "Role of sinoatrial node architecture in maintaining a balanced source-sink relationship and synchronous cardiac pacemaking," Frontiers in Physiology, vol. 5, no. 446, 2014.

[48] K. Białkowska, P. Komorowski, M. Bryszewska, and K. Miłowska, "Spheroids as a type of three-dimensional cell cultures-examples of methods of preparation and the most important application," International Journal of Molecular Sciences, vol. 21, no. 17, article 6225, 2020.

[49] M. Zanoni, F. Piccinini, C. Arienti et al., "3D tumor spheroid models for in vitro therapeutic screening: a systematic approach to enhance the biological relevance of data obtained," Scientific Reports, vol. 6, no. 1, pp. 1-11, 2016.

[50] M. W. Laschke and M. D. Menger, "Life is 3D: boosting spheroid function for tissue engineering," Trends in Biotechnology, vol. 35, no. 2, pp. 133-144, 2017.

[51] Y. Guo and W. T. Pu, "Cardiomyocyte maturation: new phase in development," Circulation Research, vol. 126, no. 8, pp. 1086-1106, 2020.

[52] X. Ma, S. Dewan, J. Liu et al., “3D printed micro-scale force gauge arrays to improve human cardiac tissue maturation and enable high throughput drug testing," Acta Biomaterialia, vol. 95, pp. 319-327, 2019.

[53] X. Ma, J. Liu, W. Zhu et al., "3D bioprinting of functional tissue models for personalized drug screening and in vitro disease modeling," Advanced Drug Delivery Reviews, vol. 132, pp. 235-251, 2018.

[54] P. Datta, M. Dey, Z. Ataie, D. Unutmaz, and I. T. Ozbolat, “3D bioprinting for reconstituting the cancer microenvironment," npj Precision Oncology, vol. 4, no. 1, pp. 1-13, 2020.

[55] J. M. Squire, "Muscle contraction: sliding filament history, sarcomere dynamics and the two Huxleys," Global cardiology science \& practice, vol. 2016, no. 2, article e201611, 2016.

[56] A. M. Chaulin, O. E. Abashina, and D. V. Duplyakov, "Pathophysiological mechanisms of cardiotoxicity in chemotherapeutic agents," Russian Open Medical Journal, vol. 9, no. 3, article e0305, 2020.

[57] L. Polonchuk, M. Chabria, L. Badi et al., "Cardiac spheroids as promising in vitro models to study the human heart microenvironment," Scientific Reports, vol. 7, no. 1, article 7005, 2017.

[58] S. Wüst, M. E. Godla, R. Müller, and S. Hofmann, “Tunable hydrogel composite with two-step processing in combination with innovative hardware upgrade for cell-based threedimensional bioprinting," Acta Biomaterialia, vol. 10, no. 2, pp. 630-640, 2014.

[59] Y. Du, E. Lo, S. Ali, and A. Khademhosseini, "Directed assembly of cell-laden microgels for fabrication of 3D tissue constructs," Proceedings of the National Academy of Sciences of the United States of America, vol. 105, no. 28, pp. 95229527, 2008. 
[60] A. Bakopoulou, A. Georgopoulou, I. Grivas et al., "Dental pulp stem cells in chitosan/gelatin scaffolds for enhanced orofacial bone regeneration," Dental Materials, vol. 35, no. 2, pp. 310$327,2019$.

[61] G. Chen and N. Kawazoe, Preparation of polymer scaffolds by ice particulate method for tissue engineering, pp. 77-95, 2016.

[62] M. Bartoš, T. Suchý, and R. Foltán, "Note on the use of different approaches to determine the pore sizes of tissue engineering scaffolds: what do we measure?," Biomedical Engineering Online, vol. 17, no. 1, p. 110, 2018.

[63] R. S. Stowers, S. C. Allen, and L. J. Suggs, "Dynamic phototuning of 3D hydrogel stiffness," Proceedings of the National Academy of Sciences of the United States of America, vol. 112, no. 7, pp. 1953-1958, 2015.

[64] A. R. Pinto, A. Ilinykh, M. J. Ivey et al., "Revisiting cardiac cellular composition," Circulation Research, vol. 118, no. 3, pp. 400-409, 2016.

[65] B. Adeniran, B. D. Bjarkadottir, R. Appeltant, S. Lane, and S. A. Williams, "Improved preservation of ovarian tissue morphology that is compatible with antigen detection using a fixative mixture of formalin and acetic acid," Human Reproduction, vol. 36, no. 7, pp. 1871-1890, 2021.

[66] A. Agarwal, Y. Farouz, A. P. Nesmith, L. F. Deravi, M. L. McCain, and K. K. Parker, "Micropatterning alginate substrates for in vitro cardiovascular muscle on a chip," Advanced Functional Materials, vol. 23, no. 30, pp. 3738-3746, 2013. 